\title{
Using Data Assimilation to Better Predict Contaminant Transport in Fluids
}

\author{
Jacob Honeycutt ${ }^{*} \quad$ Hannah Johnson ${ }^{\dagger}$ \\ Sarah Kelly ${ }^{\ddagger}$ \\ Sponsors: Leo Rebholz and Camille Zerfas
}

September 7, 2018

\begin{abstract}
We study data assimilation in fluid transport models for contaminant transport, using the recently proposed nudging technique proposed by Titi et al. in 2014 [2]. We prove, under the assumption of a sufficiently fine mesh and appropriately chosen nudging parameter, that for any initial condition, the data assimilated model solution is long-time stable and will converge to the true solution exponentially fast in time. We prove this first at the PDE level, and then also for a backward Euler - finite element discretization. Results of a numerical test are also given that illustrate our theory and show the effectiveness of the proposed method.
\end{abstract}

\section{Introduction}

Contaminants can be found in nearly every body of water across the world, and most are a byproduct of human error during the transportation of materials. For example, there have been numerous oil spills in nearly every ocean across the globe. A recent disaster was the Deepwater Horizon oil spill that occurred in the Gulf of Mexico in

${ }^{*}$ Department of Mathematical Sciences, Clemson University, Clemson, SC, 29634; email: jghoney@clemson.edu

†Department of Mathematical Sciences, Clemson University, Clemson, SC, 29634; email: hjohns6@clemson.edu

${ }_{\ddagger}^{\ddagger}$ Department of Mathematical Sciences, Clemson University, Clemson, SC, 29634; email: skelly5@clemson.edu 
2010. Nearly 53,000 barrels of oil were spilling into the Gulf of Mexico every day, and the leak was not able to be controlled for three months [5, 13]. The Deepwater Horizon oil spill can be compared to the 1989 Exxon-Valdez oil spill, which is still reaping its effect on the coastline of the Prince William Sound in Alaska. More than 11 million gallons of oil were spilled in this disaster, which damaged nearly 1,300 miles of shoreline. Almost thirty years later, the coastal ecosystem in this area is still being affected by the oil that washes up on the shorelines [14]. In the authors' own backyard, there remains a 38 year old ban on eating fish out of Lake Hartwell due to industrial pollutants from many decades ago [6].

The motivation behind this work is to better predict how contaminants, such as oil or other chemicals, disperse and spread through rivers and lakes. A critical issue that arises when formulating and calculating a prediction is that we almost never know the initial condition to a sufficient degree of accuracy. While taking an educated guess from a small amount of incomplete data might seem like a reasonable approach in this situation, use of incorrect or incomplete initial data can lead to misleading or wrong predictions, especially over longer periods of time. A better approach, which we employ here, is to incorporate into the model and calculation true data from measurements at more times than just the initial time. How to incorporate such data into a model/calculation is a current topic of research, and significant progress has been made in recent years for doing so in various fluid systems $[2,1,4,3,7,9,8,10]$.

To address this issue, we consider the incorporation of data assimilation (DA) techniques pioneered in 2014 in [2] into the equations that describe transport by a fluid. The general idea of this type of DA is to 'nudge' the model / computed solution toward data measurements, but only at the points where the measurements occur. Contaminant transport prediction suffers from having poor initial conditions in practice, as measurements can only be taken at a small number of locations. However, even though we have inexact initial data, we can have access to measurement data at certain points in space at all times. Thus, the methods of [2] seem to be a good candidate to do this in a fluid transport system. We will show mathematically that it works: we prove that over sufficiently long periods of time, the DA solution converges to the true solution, regardless of how bad the initial condition is, provided enough measurements are taken in space. We do this both at the PDE level, and for the discrete solution (the discrete solution converges only up to discretization parameters). We also give results of numerical tests that illustrate how well it can work. To our knowledge, this type of DA has not been considered for this application. 


\section{Notation and Mathematical Preliminaries}

We assume the domain $\Omega \subset \mathbb{R}^{d}$ ( $\mathrm{d}=2$ or 3 ) is convex with smooth boundary, or is a convex polygon. The boundary of the domain, $\partial \Omega$, is decomposed into two pieces, $\partial \Omega=\Gamma_{1} \cup \Gamma_{2}$, with meas $\left(\Gamma_{1} \cap \Gamma_{2}\right)=0$. We require that meas $\left(\Gamma_{1}\right)>0$.

The spaces $L^{2}(\Omega)$ and $H^{1}(\Omega)$ are defined in the usual way by

$$
\begin{aligned}
L^{2}(\Omega) & =\left\{v: \Omega \rightarrow \mathbb{R}^{d}, \int_{\Omega}|v|^{2} d x<\infty\right\} \\
H^{1}(\Omega) & =\left\{v \in L^{2}(\Omega), \int_{\Omega}|\nabla v|^{2} d x<\infty\right\} .
\end{aligned}
$$

The space $H_{0}^{1}(\Omega)$ is functions in $H^{1}(\Omega)$ that are 0 on the boundary.

The $L^{2}(\Omega)$ inner product and norm, and $H^{1}(\Omega)$ norm will be defined by

$$
\begin{aligned}
(v, w) & :=\int_{\Omega} v \cdot w d x, \\
\|v\| & :=(v, v)^{1 / 2}, \\
\|v\|_{H^{1}} & :=\left(\|v\|^{2}+\|\nabla v\|^{2}\right)^{1 / 2} .
\end{aligned}
$$

Other norms will be clearly labeled.

Define the space $X$ by

$$
X=\left\{v \in H^{1}(\Omega),\left.v\right|_{\Gamma_{1}}=0\right\} .
$$

We note that the Poincaré inequality holds in $X$ : for all $v \in X$, there exists $C_{P}$ depending only on the size of the domain, such that

$$
\|v\| \leq C_{P}\|\nabla v\| .
$$

Due to this inequality, we have that the $H^{1}$ norm is equivalent to the $L^{2}$ norm of the gradient for functions in $X$. Hence we will define

$$
\|v\|_{X}:=\|\nabla v\| .
$$

We will employ Green's theorem, which states

$$
(-\Delta v, w)=(\nabla v, \nabla w)-\int_{\partial \Omega}(\nabla v \cdot n) \cdot w d s
$$


for a piecewise smooth, positively oriented, simple, closed curve in a plane, $\partial \Omega$, and functions with continuous partial derivatives, $v$ and $w$.

We will utilize the following property of the convection term that arises in fluid transport: For $U \in H_{0}^{1}(\Omega)$ with $\nabla \cdot U=0$, and $v \in X$, we have that [11]

$$
(U \cdot \nabla v, v)=0
$$

Several inequalities important in this work are known to hold in $X$. The first of these inequalities is the Cauchy-Schwarz inequality which states that for vectors $u$ and $v$ in an inner product space equipped with inner product $\langle\cdot, \cdot\rangle$, it holds that

$$
|<u, v>| \leq\|u\|\|v\|
$$

Young's inequality states that for $p, q$ as strictly positive real numbers satisfying $\frac{1}{p}+\frac{1}{q}=1$ then for any nonnegative real numbers $a$ and $b$,

$$
a b \leq \frac{a^{p}}{p}+\frac{b^{q}}{q}
$$

We will also use Hölder's inequality which states that for functions $f, g$ and $h$, and strictly positive real numbers $p, q$ and $r$, where $\frac{1}{p}+\frac{1}{q}+\frac{1}{r}=1$,

$$
\|f g h\|_{1} \leq\|f\|_{p}\|g\|_{q}\|h\|_{r}
$$

We will also use the polarization identity, which states that for functions $u, v \in X$,

$$
(u-v, u)=\frac{1}{2}\left(\|u\|^{2}-\|v\|^{2}+\|u-v\|^{2}\right) .
$$

\section{The fluid transport equation and data assimila- tion}

The equation we use to model the transport of a contaminant $c(x, t)$ in an incompressible fluid moving with velocity $U(x) \in H_{0}^{1}(\Omega)$ is given by:

$$
\begin{aligned}
c_{t}-\epsilon \Delta c+U \cdot \nabla c & =0, \\
c(x, 0) & =c_{0}(x),
\end{aligned}
$$


where $\epsilon>0$ is a physical constant representing the diffusion rate, and $c_{0}$ is the initial condition. Appropriate boundary conditions must also be defined, and we will use

$$
\left.c\right|_{\Gamma_{1}}=0,\left.\quad \nabla c \cdot n\right|_{\Gamma_{2}}=0,
$$

where $n$ is the outward unit normal to $\Omega$. The boundary condition on $\Gamma_{1}$ can be physically justified if one moves far enough away from a spill, and the spill is moving in the opposite direction from $\Gamma_{1}$. The condition on $\Gamma_{2}$ is the same as one finds for temperature transport, and corresponds to the transport not being affected by the boundary.

DA is used to merge observational data with a model that accurately forecasts the state of a physical system. The type of DA we consider follows [2], and adds a simple penalty to the system above, to force the solution to be closer to the (3.1)(3.2) solution, but only at a small number of points, referred to as measurement points. The idea is that if we nudge the system toward the true solution (assuming measurements and model are correct), then the entire model solution should be moved toward the true solution. To extract these points, we use an interpolation operator $I_{h}$, and require that $I_{h}$ has the interpolation property: for all $v \in H^{1}(\Omega)$, there exists a constant $C_{I}$ such that

$$
\left\|I_{h}(v)-v\right\| \leq C_{I} h\|\nabla v\|
$$

where $C_{I}$ is independent of $h$, and $h$ represents a spacing width of the measurement points. For example, one can think of the interpolation operator as a piecewise constant interpolation onto a triangulation of the domain.

The DA system we consider for improving predictions of (3.1)-(3.2) takes the form

$$
\begin{aligned}
\tilde{c}_{t}-\epsilon \Delta \tilde{c}+U \cdot \nabla \tilde{c}+\mu\left(I_{h}(\tilde{c}-c)\right) & =0, \\
\tilde{c}(x, 0) & =0,
\end{aligned}
$$

along with the same Dirichlet / Neumann boundary conditions for the system above. This system is a modification of (3.1)-(3.2), with the nudging term $\mu\left(I_{h}(\tilde{c}-c)\right)$ added to the left hand side, where $\mu>0$ is a penalty parameter. We note that the initial condition is taken to be 0 for simplicity, but could be arbitrary in $L^{2}(\Omega)$.

The well-posedness and regularity theory for (3.3)-(3.4) is outside the scope of this work. We will assume that weak solutions exist uniquely in $X$ for both systems [defined in the next section]. Well-posedness and regularity properties of (3.3)-(3.4) are inherited from (3.1)-(3.2), as DA nudging term will preserve coercivity from (3.1)- 
(3.2), provided $h$ is sufficiently small. This can be seen from the following calculation, which uses Cauchy Schwarz and interpolation properties, followed by Young's inequality:

$$
\begin{aligned}
\mu\left(I_{h}(v), v\right) & =\mu\|v\|^{2}-\mu\left(v-I_{h}(v), v\right) \\
& \geq \mu\|v\|^{2}-\mu\left\|v-I_{h}(v)\right\|\|v\| \\
& \geq \mu\|v\|^{2}-\mu C_{I} h\|\nabla v\|\|v\|, \\
& \geq \frac{\mu}{2}\|v\|^{2}-\frac{\mu}{2} C_{I}^{2} h^{2}\|\nabla v\|^{2} .
\end{aligned}
$$

This implies that for $h$ sufficiently small, the DA nudging term will preserve coercivity from (3.1)-(3.2), and thus inherit its solution properties.

\subsection{Convergence of the DA system to the true solution as $t \rightarrow \infty$.}

We now prove that the weak solution of (3.3)-(3.4) converges to the weak solution of (3.1)-(3.2), assuming $\mu$ and $h$ are chosen appropriately. To do this, we first define weak solutions, which are obtained by testing the systems above with arbitrary $v \in X$. As noted above, solutions to these weak systems are known to exist uniquely.

For the fluid transport equation, we wish to find $c \in X \times[0, T)$ satisfying for all $v \in X$,

$$
\begin{aligned}
\left(c_{t}, v\right)+\epsilon(\nabla c, \nabla v)+(U \cdot \nabla c, v) & =0,(t>0) \\
(c(0), v) & =\left(c_{0}, v\right) .
\end{aligned}
$$

This is the model that we accept as the true solution. However, since we cannot know $c_{0}$ exactly, we cannot solve this model, which is why we consider herein incorporating DA.

For the DA system, we wish to find $\tilde{c} \in X \times[0, T)$ satisfying for all $v \in X$,

$$
\begin{gathered}
\left(\tilde{c}_{t}, v\right)+\epsilon(\nabla \tilde{c}, \nabla v)+(U \cdot \nabla \tilde{c}, v)+\mu\left(I_{h}(\tilde{c}-c), v\right)=0, \quad(t>0) \\
\tilde{c}(0)=0 .
\end{gathered}
$$

We have chosen 0 to be the initial condition of the DA system, however for the theory that follows it could be chosen as any arbitrary $L^{2}(\Omega)$ function. A zero initial condition corresponds to having no knowledge of where the contaminant is at the 
start time.

Theorem 1. Suppose $\epsilon-\frac{\mu}{2} C_{I}^{2} h^{2} \geq 0$ (this is always possible with sufficiently small $h$ ). Then $\tilde{c}$ converges to $c$ exponentially fast as $t$ increases. In particular,

$$
\|c-\tilde{c}\| \leq\left\|c_{0}\right\| \exp (-\mu t)
$$

Remark 1. This theorem proves that for $h$ sufficiently small, a nudging parameter $\mu$ can be found that will force the DA solution to converge to the true solution, exponentially fast. Moreover, the smaller $h$ is, the larger $\mu$ can be chosen, which will allow for faster convergence.

Proof. Subtracting (3.7) from (3.5) gives the error equation, for all $v \in X$,

$$
\left(e_{t}, v\right)+\epsilon(\nabla e, \nabla v)+(U \cdot \nabla e, v)+\mu\left(I_{h}(e), v\right)=0,(t>0),
$$

where $e:=c-\tilde{c}$. Choosing $v=e$, which is permitted since $c, \tilde{c} \in X$, we obtain

$$
\frac{1}{2} \frac{d}{d t}\|e\|^{2}+\epsilon\|\nabla e\|^{2}+(U \cdot \nabla e, e)+\mu\left(I_{h}(e), e\right)=0
$$

Now since $(U \cdot \nabla e, e)=0$, the equation reduces to

$$
\frac{1}{2} \frac{d}{d t}\|e\|^{2}+\epsilon\|\nabla e\|^{2}+\mu\left(I_{h}(e), e\right)=0 .
$$

Adding and subtracting $e$ to $I_{h}(e)$, we can write the equation as

$$
\frac{1}{2} \frac{d}{d t}\|e\|^{2}+\epsilon\|\nabla e\|^{2}+\mu\|e\|^{2}=-\mu\left(I_{h}(e)-e, e\right) .
$$

Applying Cauchy-Schwarz and interpolation properties on the right hand side, we obtain the bound

$$
\frac{1}{2} \frac{d}{d t}\|e\|^{2}+\epsilon\|\nabla e\|^{2}+\mu\|e\|^{2} \leq \mu\left\|I_{h}(e)-e\right\|\|e\| \leq C_{I} h \mu\|\nabla e\|\|e\| .
$$

Young's inequality implies that

$$
\mu C_{I} h\|\nabla e\|\|e\| \leq \frac{\mu}{2}\|e\|^{2}+\frac{\mu}{2} C_{I}^{2} h^{2}\|\nabla e\|^{2},
$$

and so using this in the bound above, and multiplying both sides by 2 , we obtain

$$
\frac{d}{d t}\|e\|^{2}+2\left(\epsilon-\frac{\mu}{2} C_{I}^{2} h^{2}\right)\|\nabla e\|^{2}+\mu\|e\|^{2} \leq 0
$$


Using the assumptions on $\mu$ and $h$, the second term is non-negative, and so we can drop it, leaving

$$
\frac{d}{d t}\|e\|^{2}+\mu\|e\|^{2} \leq 0
$$

We can solve this differential inequality using an integrating factor of $\exp (\mu t)$, which yields

$$
\|e(t)\| \leq\|e(0)\| \exp (-\mu t)=\left\|c_{0}\right\| \exp (-\mu t) .
$$

This completes the proof.

\section{Discretization and Numerical Analysis}

In this section, we consider numerical algorithms for the systems above. We first introduce the necessary notation and inequalities needed for the numerical analysis. We then propose finite element algorithms to approximate solutions to the systems (3.5)-(3.6) and (3.7)-(3.8). Finally, we prove that the solutions to the DA algorithm converge to the solutions of the discrete transport algorithm.

\subsection{Discretization Preliminaries}

In our discrete setting, a mesh $\tau_{h}$ denotes a conforming triangulation of $\Omega$ with diameter $h$. The finite dimensional space $X_{h} \subset X$ is defined to be:

$$
X_{h}=P_{k}\left(\tau_{h}\right) \cap X
$$

where $P_{k}\left(\tau_{h}\right)$ represents the space of piecewise degree $k$ polynomials.

We continue to assume our interpolant $I_{h}$ for the DA algorithm satisfies the following inequality:

$$
\left\|I_{h}(\phi)-\phi\right\| \leq C_{I} h\|\nabla \phi\|
$$

for all $\phi \in X$, where $\mathrm{h}$ is a point spacing for the interpolant, proportional to $\frac{1}{\sqrt{N}}$, where $N$ is the number of points used by the interpolant and $C_{I}$ is an interpolation constant. We note this $h$ need not be as fine as the mesh width, but the inequality (4.1) must hold without $C_{I}$ being "too large". 


\subsection{Numerical Algorithms}

We now propose a discrete finite element algorithm for the model (3.5)-(3.6) that uses a backward Euler approximation to the time derivative. It is given by the following algorithm:

Algorithm 1. Given initial condition $c_{h}^{0}$, end time $T$, time step size $\Delta t>0$, and a fluid velocity $U \in H_{0}^{1}(\Omega)$ with $\nabla \cdot U=0$, find $c_{h}^{n+1} \in X_{h}$ for $\mathrm{n}=0,1,2, \ldots, \frac{T}{\Delta t}=M$ such that

$$
\frac{1}{\Delta t}\left(c_{h}^{n+1}-c_{h}^{n}, v_{h}\right)+\epsilon\left(\nabla c_{h}^{n+1}, \nabla v_{h}\right)+\left(U \cdot \nabla c_{h}^{n+1}, v_{h}\right)=0
$$

for all $v_{h} \in X_{h}$.

Similarly, the finite element, backward Euler data assimilation algorithm is given as follows.

Algorithm 2. Given initial condition $\tilde{c}_{h}^{0}=0$, end time $T$, time step size $\Delta t>0$, true solution at measurement points $I_{h}\left(c_{h}^{n}\right)$, and a fluid velocity $U \in H_{0}^{1}(\Omega)$ with $\nabla \cdot U=0$, find $\tilde{c}_{h}^{n+1} \in X_{h}$ for $\mathrm{n}=0,1,2, \ldots, \frac{T}{\Delta t}=M$ such that

$$
\frac{1}{\Delta t}\left(\tilde{c}_{h}^{n+1}-\tilde{c}_{h}^{n}, v_{h}\right)+\epsilon\left(\nabla \tilde{c}_{h}^{n+1}, \nabla v_{h}\right)+\left(U \cdot \nabla \tilde{c}_{h}^{n+1}, v_{h}\right)+\mu\left(I_{h}\left(\tilde{c}_{h}^{n+1}-c_{h}^{n+1}\right), v_{h}\right)=0 .
$$

\subsection{Well-posedness of the algorithms}

An algorithm is well-posed if solutions exist uniquely and are bounded continuously by the data. We prove here that solutions exist uniquely for both of the algorithms above. We consider first the well-posedness and long-time stability (bounded at all times) of Algorithm 1.

Theorem 2. Algorithm 1 is well-posed and solutions are long-time stable.

Proof. Since this is a time stepping algorithm with a given initial condition, it suffices to prove well-posedness of an arbitrary time step. Thus consider time step $n$, with $c_{h}^{n}$ given uniquely. Suppose there are two solutions, $c_{1}$ and $c_{2}$. We will begin by subtracting (4.2) using $c_{1}$ and $c_{2}$, which yields

$$
\frac{1}{\Delta t}\left(c_{1}-c_{2}, v_{h}\right)+\epsilon\left(\nabla\left(c_{1}-c_{2}\right), \nabla v_{h}\right)+\left(U \cdot \nabla\left(c_{1}-c_{2}\right), v_{h}\right)=0
$$


Now we will let $v_{h}=c_{1}-c_{2}$ to get

$$
\frac{1}{\Delta t}\left\|c_{1}-c_{2}\right\|^{2}+\epsilon\left\|\nabla\left(c_{1}-c_{2}\right)\right\|^{2}+\left(U \cdot \nabla\left(c_{1}-c_{2}\right), c_{1}-c_{2}\right)=0
$$

Using properties of the convective term discussed in section $2,\left(U \cdot \nabla\left(c_{1}-c_{2}\right), c_{1}-c_{2}\right)=$ 0 . Thus we get that

$$
\frac{1}{\Delta t}\left\|c_{1}-c_{2}\right\|^{2}+\epsilon\left\|\nabla\left(c_{1}-c_{2}\right)\right\|^{2}=0
$$

This implies that $c_{1}=c_{2}$ and thus solutions at time step $n$ are unique. But since (4.2) is linear and finite dimensional, uniqueness implies existence, and thus we have solutions at time step $n$ exist uniquely.

Lastly, we need to show solutions to Algorithm 1 are bounded continuously by the data. Choosing $v_{h}=c_{h}^{n+1}$ in (4.2), we obtain

$$
\frac{1}{2 \Delta t}\left(\left\|c_{h}^{n+1}\right\|^{2}-\left\|c_{h}^{n}\right\|^{2}+\left\|c_{h}^{n+1}-c_{h}^{n}\right\|^{2}\right)+\epsilon\left\|\nabla c_{h}^{n+1}\right\|^{2}=0
$$

Dropping the third term on the left hand side (as it is non-negative), multiplying both sides by $2 \Delta t$ and summing over time steps gives

$$
\left\|c_{h}^{n}\right\|^{2}+2 \epsilon \Delta t \sum_{j=1}^{n}\left\|\nabla c_{h}^{j}\right\|^{2} \leq\left\|c_{h}^{0}\right\|^{2} .
$$

This implies the solution is continuously bounded by the data, which is just the initial condition.

To prove long-time stability, starting from (4.4), we have

$$
\left\|c_{h}^{n+1}\right\|^{2}+2 \epsilon \Delta t\left\|\nabla c_{h}^{n+1}\right\|^{2} \leq\left\|c_{h}^{n}\right\|^{2}
$$

Now using the Poincaré inequality, we obtain

$$
\left\|c_{h}^{n+1}\right\|^{2}\left(1+2 \epsilon \Delta t C_{p}^{-2}\right) \leq\left\|c_{h}^{n}\right\|^{2}
$$

This implies $\left\|c_{h}^{n}\right\| \leq\left\|c_{h}^{0}\right\|$ for any $\mathrm{n}$, which means the solution is long-time stable.

The DA algorithm is also well-posed, which we prove next. It is also long-time stable, which follows from Theorem 3 below, since this theorem proves the DA algorithm solution converges exponentially fast in time to a long-time stable solution. 
Lemma 1. Algorithm 2 is well-posed provided $\epsilon-\frac{C_{I}^{2} h^{2} \mu}{2} \geq 0$.

Proof. We proceed in a fashion similar to the previous proof. Assume two solutions at time step $n$, call them $c_{1}$ and $c_{2}$, and subtract the two equations that they satisfy, i.e. (4.3), to obtain

$$
\frac{1}{\Delta t}\left\|c_{1}-c_{2}\right\|^{2}+\epsilon\left\|\nabla\left(c_{1}-c_{2}\right)\right\|^{2}+\mu\left(I_{h}\left(c_{1}-c_{2}\right), c_{1}-c_{2}\right)=0
$$

Adding and subtracting $\left(c_{1}-c_{2}\right)$ in the last term, we get

$$
\left(\frac{1}{\Delta t}+\mu\right)\left\|c_{1}-c_{2}\right\|^{2}+\epsilon\left\|\nabla\left(c_{1}-c_{2}\right)\right\|^{2}=-\mu\left(I_{h}\left(c_{1}-c_{2}\right)-\left(c_{1}-c_{2}\right), c_{1}-c_{2}\right)
$$

Applying Cauchy-Schwarz and interpolation properties to the right hand side, we find that

$$
\left(\frac{1}{\Delta t}+\mu\right)\left\|c_{1}-c_{2}\right\|^{2}+\epsilon\left\|\nabla\left(c_{1}-c_{2}\right)\right\|^{2} \leq \mu C_{I} h\left\|\nabla\left(c_{1}-c_{2}\right)\right\|\left\|c_{1}-c_{2}\right\| .
$$

Now with Young's inequality on the right hand side, we get the bound

$$
\left(\frac{1}{\Delta t}+\frac{\mu}{2}\right)\left\|c_{1}-c_{2}\right\|^{2}+\left(\epsilon-\frac{C_{I}^{2} h^{2} \mu}{2}\right)\left\|\nabla\left(c_{1}-c_{2}\right)\right\|^{2} \leq 0
$$

Thus using the assumption on $h$ and $\mu$, we get that $c_{1}=c_{2}$. From here, well-posedness follows as in the proof above.

\subsection{Convergence of the DA algorithm}

We will now prove that the solutions to the data assimilation algorithm converge to the solutions of Algorithm 1 as long as the stepsize $h$ is sufficiently small.

Theorem 3. Provided $\mu \leq \frac{2 \epsilon}{h^{2} C_{I}^{2}}$, the solutions to Algorithm 2 converge to the solutions of Algorithm 1 as $n \rightarrow \infty$. That is,

$$
\left\|c_{h}^{n}-\tilde{c}_{h}^{n}\right\|^{2} \leq(1+\mu \Delta t)^{-n}\left\|c_{h}^{0}\right\|^{2}
$$

Proof. Begin by subtracting equation (4.3) from (4.2) and setting $e^{n}:=c_{h}^{n}-\tilde{c}_{h}^{n}$ to obtain the difference equation

$$
\frac{1}{\Delta t}\left(e^{n+1}-e^{n}, v_{h}\right)+\left(U \cdot \nabla e^{n+1}, v_{h}\right)+\epsilon\left(\nabla e^{n+1}, \nabla v_{h}\right)+\mu\left(I_{h}\left(e^{n+1}\right), v_{h}\right)=0
$$


Next, choose $v_{h}=e^{n+1}$, which is possible since $e^{n+1} \in X_{h}$ since both $c_{h}^{n+1}, \tilde{c}_{h}^{n+1} \in X_{h}$, which yields with the polarization identity,

$$
\begin{aligned}
\frac{1}{2 \Delta t}\left[\left\|e^{n+1}\right\|^{2}-\left\|e^{n}\right\|\left\|^{2}+\right\| e^{n+1}-e^{n} \|^{2}\right] & +\epsilon\left\|\nabla e^{n+1}\right\|^{2} \\
& +\mu\left\|e^{n+1}\right\|^{2}=-\mu\left(I_{h}\left(e^{n+1}\right)-e^{n+1}, e^{n+1}\right),
\end{aligned}
$$

where we have applied the polarization identity to the time derivative term and added and subtracted $e^{n+1}$ to the first argument of our interpolation term. To bound the right hand side term of the error equation, we apply Cauchy-Schwarz then Young's inequality and interpolation properties to get

$$
\begin{aligned}
\mu\left|\left(I_{h}\left(e^{n+1}\right)-e^{n+1}, e^{n+1}\right)\right| & \leq \mu\left\|I_{h} e^{n+1}-e^{n+1}\right\|\left\|e^{n+1}\right\| \\
& \leq \mu C_{I} h\left\|\nabla e^{n+1}\right\|\left\|e^{n+1}\right\| \\
& \leq \mu\left(\frac{C_{I}^{2} h^{2}}{2}\left\|\nabla e^{n+1}\right\|^{2}+\frac{1}{2}\left\|e^{n+1}\right\|^{2}\right) \\
& =\frac{\mu C_{I}^{2} h^{2}}{2}\left\|\nabla e^{n+1}\right\|^{2}+\frac{\mu}{2}\left\|e^{n+1}\right\|^{2} .
\end{aligned}
$$

Thus, error equation bound reduces to

$$
\frac{1}{2 \Delta t}\left[\left\|e^{n+1}\right\|^{2}-\left\|e^{n}\right\|^{2}+\left\|e^{n+1}-e^{n}\right\|^{2}\right]+\left(\epsilon-\frac{\mu C_{I}^{2} h^{2}}{2}\right)\left\|\nabla e^{n+1}\right\|^{2}+\frac{\mu}{2}\left\|e^{n+1}\right\|^{2} \leq 0 .
$$

Now using the assumption on $\mu$ and $h$, dropping nonnegative terms on the left hand side, and reducing produces the bound

$$
(1+\Delta t \mu)\left\|e^{n+1}\right\|^{2} \leq\left\|e^{n}\right\|^{2}
$$

which can also be written as:

$$
\begin{aligned}
\left\|e^{n+1}\right\|^{2} & \leq(1+\mu \Delta t)^{-1}\left\|e^{n}\right\|^{2} \\
& \leq(1+\mu \Delta t)^{-2}\left\|e^{n-1}\right\|^{2} \\
& \vdots \\
& \leq(1+\mu \Delta t)^{-(n+1)}\left\|e^{0}\right\|^{2} \\
& =(1+\mu \Delta t)^{-(n+1)}\left\|c_{h}^{0}\right\|^{2} .
\end{aligned}
$$




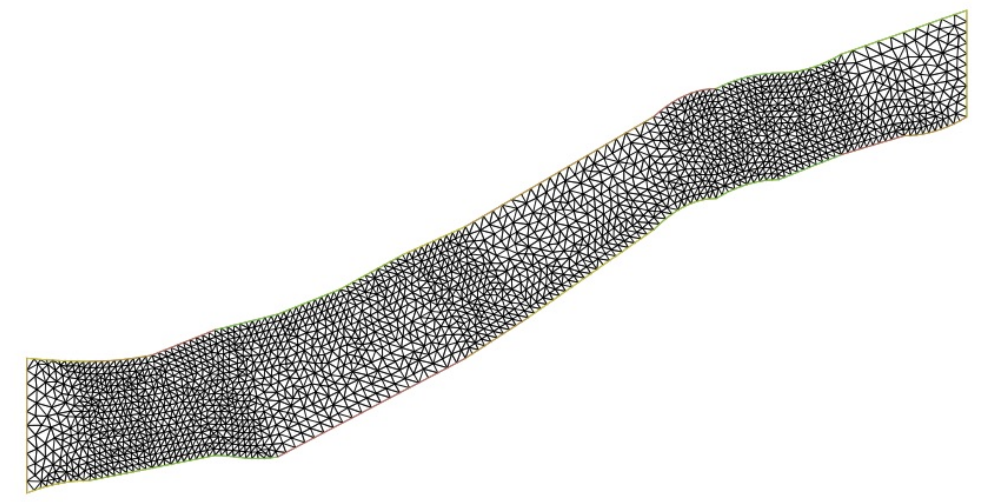

Figure 1: Shown above is a mesh of the domain (river).

Thus, $e^{n}$ converges to 0 exponentially fast as $n \rightarrow \infty$. Therefore, the data assimilation algorithm solution converges to the true model discrete algorithm solution as $n \rightarrow$ $\infty$.

\section{$5 \quad$ Numerical Tests}

We now perform a numerical test, to illustrate the theory of the previous section. The domain for this test was derived from the Holston River, which flows through Virginia and Tennessee. Located beside the Holston River is an ammunition plant, which is believed to have caused major pollution in the Holston River [12]. Our goal is to use Algorithm 2 to produce an accurate prediction of contaminant transport despite poor initial condition data.

First, we define a velocity field by finding a solution to the Stokes problem with unit viscosity, a parabolic inlet profile on the left side of the domain (with max velocity 1), a zero-traction outflow, and no-slip walls. This was solved in freefem using TaylorHood elements and the mesh shown in figure 1. We refer to [11] for details of the finite element algorithm for Stokes. This produces the $U$ velocity in the river, which is a rather smooth velocity field flowing from left to right on the domain.

We then use this $U$ to solve Algorithm 1 and Algorithm 2, and for Algorithm 2 we nudge toward the solution to Algorithm 1 at each time step. An end time $\mathrm{T}=1$ was used, along with time step size $\Delta t=0.002$ and nudging parameter $\mu=1$. We use degree $P_{3}$ elements for both algorithms, and for the interpolation we use $P_{0}$ elements on the same mesh. The initial condition for Algorithm for 4.2 was 0, and the initial condition for Algorithm 4.1 was three small blobs of contaminant, as shown in figure 2. 
Figure 2 shows the progression of the two algorithms. We observe that by 0.226 time units the DA solution is very close to the true model solution, and by 0.526 time units they are visually indistinguishable. The differences between the two solutions in the $L^{2}$ norm is plotted in figure 3 , both on the usual scale and on a semilogy scale. The fact the the semilogy plot is linear is in perfect agreement with our theory above, which says that the convergence is exponentially fast.

True solution

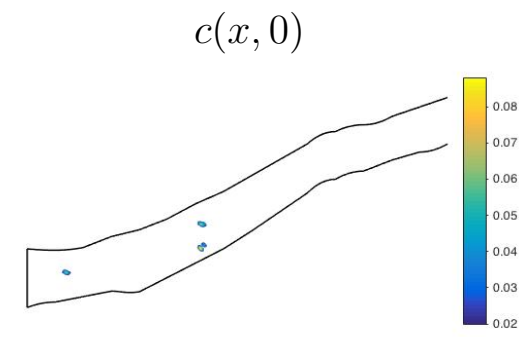

$c(x, 0.226)$

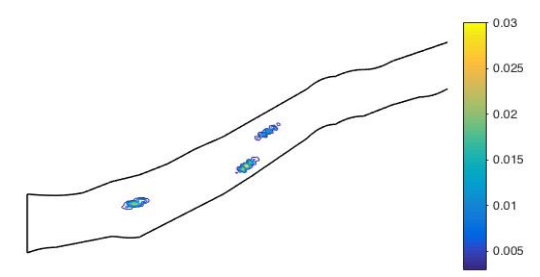

$c(x, 0.526)$

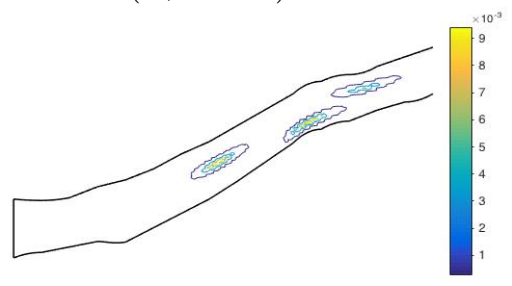

$c(x, 0.826)$

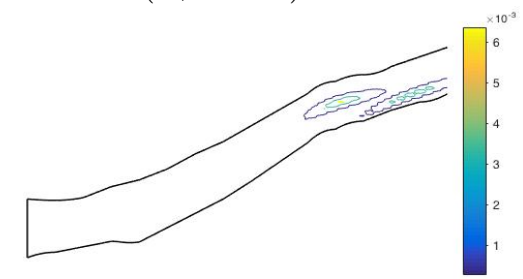

Data Assimilated solution

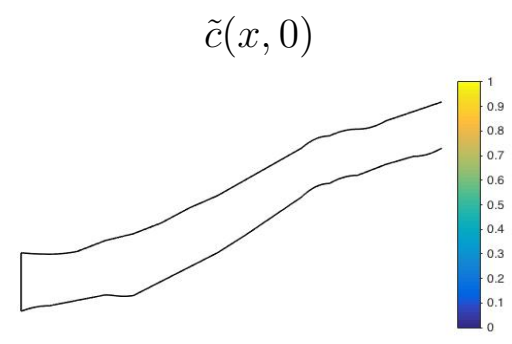

$\tilde{c}(x, 0.226)$

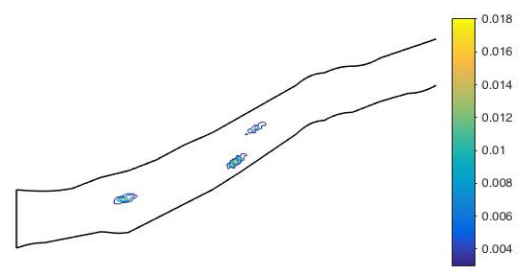

$\tilde{c}(x, 0.526)$

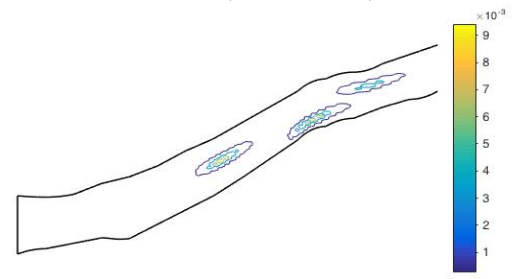

$\tilde{c}(x, 0.826)$

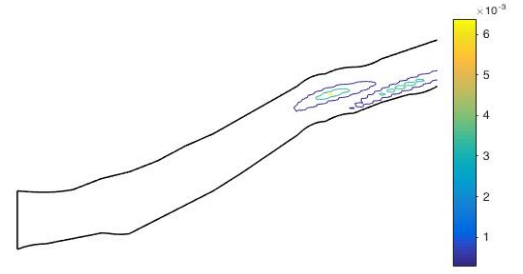

Figure 2: The data assimilated solution approaches the true solution over time. 

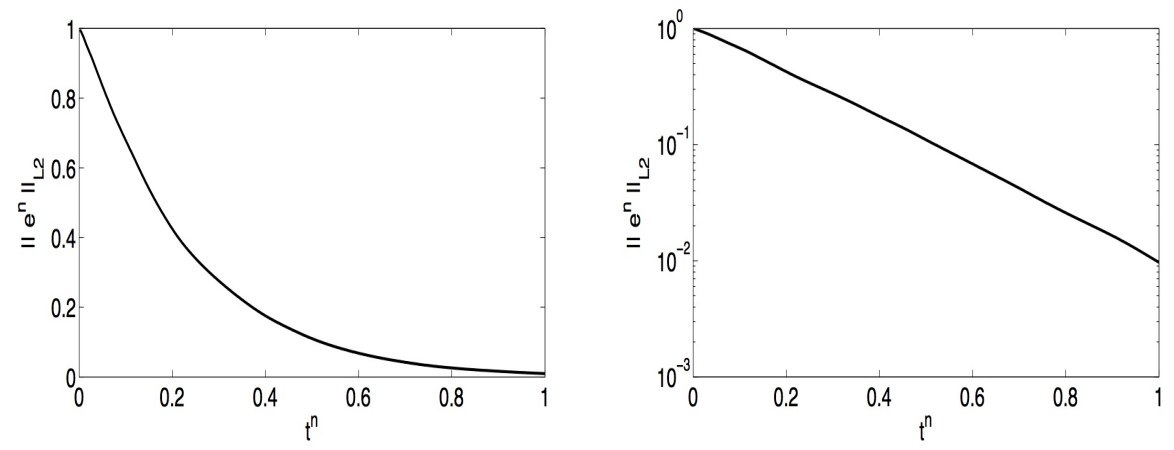

Figure 3: Convergence in time for the DA solution to the true model solution. On the left is the usual scale, and on the right is the semilogy scale.

\section{Conclusions}

We have studied a DA model and algorithm for improving prediction of contaminant transport in fluids. The theory we have shown predicts that poor initial data can be overcome with the use of nudging, provided $\mu$ and $h$ are chosen appropriately. Our numerical results back up the theory in a fairly realistic setting. Future work could include testing how sensitive the convergence is to the choices of $h$ and $\mu$.

\section{References}

[1] H. Lopes A. Albanez and E. Titi. Continuous data assimilation for the threedimensional Navier-Stokes- $\alpha$ model. Asymptotic Anal., 97(1-2):139-164, 2016.

[2] A. Azouani, E. Olson, and E. Titi. Continuous data assimilation using general interpolant observables. J. Nonlinear Sci., 24(2):277-304, 2014.

[3] H. Bessaih, E. Olson, and E. Titi. Continuous data assimilation with stochastically noisy data. Nonlinearity, 28(3):729-753, 2015.

[4] A. Biswas, J. Hudson, A. Larios, and Y. Pei. Continuous data assimilation for the magneto-hydrodynamic equations in 2D using one component of the velocity and magnetic fields. Asymptotic Analysis, In press, 2017.

[5] D. Griffin N. Black and C. Devine. 5 years after the Gulf oil spill: What we do (and don't) know. CNN online, April 20, 2015.

[6] N. Cary. Eating fish from Upstate's waters poses a health hazard. Greenville News, July 10, 2014. 
[7] A. Farhat, M. Jolly, and E. Titi. Continuous data assimilation for the 2D bénard convection through velocity measurements alone. Physica D, 303:59-66, 2015.

[8] A. Farhat, E. Lunasin, and E.S. Titi. Data assimilation algorithm for 3D bénard convection in porous media employing only temperature measurements. Journal of Mathematical Analysis and Applications, 438(1):492-506, 2016.

[9] A. Farhat, E. Lunasin, and E.S. Titi. On the Charney conjecture of data assimilation employing temperature measurements alone: the paradigm of 3D planetary geostrophic model. Mathematics of Climate and Weather Forecasting, 2(1):61$74,2016$.

[10] A. Farhat, E. Lunasin, and E.S. Titi. Continuous data assimilation for a 2D bénard convection system through horizontal velocity measurements alone. Journal of Nonlinear Science, 27:1065-1087, 2017.

[11] W. Layton. An Introduction to the Numerical Analysis of Viscous Incompressible Flows. SIAM, Philadelphia, 2008.

[12] R. Lucas. WSLS 10 investigates pollution at the Radford Army Ammunition Plant. https://www.wsls.com/news/wsls-10-investigates-pollution-at-the-radfordarmy-ammunition-plant, 2016.

[13] R. Streitfeld. Panel calls for drastic steps to stop future deepwater oil spills. CNN online, January 11, 2011.

[14] A. Taylor. The Exxon Valdez oil spill: 25 years ago today. The Atlantic, March $24,2014$. 